Acknowledgements: Authors thank all the subjects, investigators and study site staff who participated in ECLIPSE.

\title{
References
}

Brightling CE, McKenna S, Hargadon B, et al. Sputum eosinophilia and the short term response to inhaled mometasone in chronic obstructive pulmonary disease. Thorax 2005; 60: 193-198.

2 Brightling CE, Monteiro W, Ward R, et al. Sputum eosinophilia and short-term response to prednisolone in chronic obstructive pulmonary disease: a randomised controlled trial. Lancet 2000; 356: 1480-1485.

3 Leigh R, Pizzichini MM, Morris MM, et al. Stable COPD: predicting benefit from high-dose inhaled corticosteroid treatment. Eur Respir J 2006; 27: 964-971.

4 Pizzichini E, Pizzichini MM, Gibson P, et al. Sputum eosinophilia predicts benefit from prednisone in smokers with chronic obstructive bronchitis. Am J Respir Crit Care Med 1998; 158: 1511-1517.

Bafadhel M, McKenna S, Terry S, et al. Acute exacerbations of chronic obstructive pulmonary disease: identification of biologic clusters and their biomarkers. Am J Respir Crit Care Med 2011; 184: 662-671.

6 Papi A, Luppi F, Franco F, et al. Pathophysiology of exacerbations of chronic obstructive pulmonary disease. Proc Am Thorac Soc 2006; 3: 245-251.

7 Siva R, Green RH, Brightling CE, et al. Eosinophilic airway inflammation and exacerbations of COPD: a randomised controlled trial. Eur Respir J 2007; 29: 906-913.

8 Bafadhel M, McKenna S, Terry S, et al. Blood eosinophils to direct corticosteroid treatment of exacerbations of chronic obstructive pulmonary disease: a randomized placebo-controlled trial. Am J Respir Crit Care Med 2012; 186: 48-55.

9 Vestbo J, Anderson W, Coxson HO, et al. Evaluation of COPD Longitudinally to Identify Predictive Surrogate Endpoints (ECLIPSE). Eur Respir J 2008; 31: 869-873.

10 Dickens JA, Miller BE, Edwards LD, et al. COPD association and repeatability of blood biomarkers in the ECLIPSE cohort. Respir Res 2011; 12: 146.

11 Gietema HA, Müller NL, Fauerbach PV, et al. Quantifying the extent of emphysema: factors associated with radiologists' estimations and quantitative indices of emphysema severity using the ECLIPSE cohort. Acad Radiol 2011; 18: 661-671.

12 Rutgers SR, Timens W, Kaufmann HF, et al. Comparison of induced sputum with bronchial wash, bronchoalveolar lavage and bronchial biopsies in COPD. Eur Respir J 2000; 15: 109-115.

13 Corren J. Inhibition of interleukin-5 for the treatment of eosinophilic diseases. Discov Med 2012; 13: 305-312.

\section{Measuring improvement in dyspnoea: should absolute or relative values be used?}

To the Editor:

The same patient outcome data from clinical trial results, when presented as absolute or relative changes, may appear different in magnitude. Recommendations are to report both absolute and relative, or at least baseline, data from which to calculate absolute values $[1,2]$. A systematic review of efficacy trials demonstrated that only relative values were reported in most study abstracts (88\%) and the main text (75\%) [3].

To inform clinical practice, outcome improvements, whether relative or absolute, must be statistically significant and clinically meaningful. A minimal clinically important difference (MCID) should inform sample size calculations for clinical trials.

Two main methods identify an MCID (distribution and anchor-based methods); ideally used together to interpret one in the context of the other [4]. The distribution method is a statistical calculation based on the baseline variability of the measure in the population studied. This gives an effect size (change after intervention divided by standard deviation of baseline scores), the magnitude of which relates to a small, moderate or large clinical effect [5]. Thus the distribution method can only be used to calculate an absolute MCID as there is no standard deviation of baseline score for a relative measure.

The anchor-based method relates the change in score to another patient-rated effect (e.g. relief score, function, or global impression of change). The anchor-based method can be used to calculate the relative MCID.

Debate surrounds whether the MCID for symptoms (e.g. pain or breathlessness) should be based on absolute or relative measures. Measures may include $0-100 \mathrm{~mm}$ visual analogue scale (VAS) or $0-10$ numerical rating scale (NRS) (0 NRS is no symptom and 10 NRS or $100 \mathrm{~mm}$ VAS is the worst imaginable symptom) for each aspect of a symptom. An absolute difference of $10 \mathrm{~mm}$ VAS may be perceived as a larger 
effect if baseline intensity was $30 \mathrm{~mm}$ (33\% relative reduction), than a baseline intensity of $90 \mathrm{~mm}(11 \%$ relative reduction) [6].

In studies of chronic breathlessness, absolute or relative differences are used in sample size calculations. Clinically meaningful relative differences are still consensus based, varying between $10 \%$ and $25 \%[7,8]$, although the absolute MCID for chronic breathlessness using the distribution and patient anchor-based methods have been calculated from pooled patient data [9]. Using the same dataset, and with respect to assessments of breathlessness intensity, this current analysis investigates: 1) whether the variability of the difference from baseline is more stable for absolute or relative measures; and 2) the patient anchor-based method calculated relative MCID.

This study analysed anonymised individual patient data pooled from four clinical trials of oral opioids for the management of breathlessness (three randomised control trials (RCT) and one observational study) as previously described [7, 10-12]. A total of 213 sets of data from 178 participants allowed for the calculation of effect size.

National Health Service ethical permission was not required for pooling anonymised data for secondary analyses. Appropriate ethics approval and written informed consent by participants had been obtained for contributing studies.

The relationship between end of intervention and baseline breathlessness intensity were plotted. To check whether variability was related to magnitude, the relationship of end-of-intervention minus baseline intensity with baseline intensity was examined, firstly for absolute and then for relative values. The relationships between these measures and baseline, and the pattern of variability of responses according to baseline intensity were displayed graphically.

The patient anchor-based method for calculating MCID, using absolute measures, was previously reported [9]. In the current communication, we use the same methods to calculate the MCID expressed as a relative value. At the end of the three placebo-controlled crossover RCTs, participants provided a blinded choice for their preferred arm. Participants' perceptions of change in breathlessness intensity, expressed as mean ratios, were examined in relation to the preferred study arm (opioid, placebo or neither). We found the ratio in the preferred arm and compared preferences for drug or placebo, repeating this for the other arm. This allowed comparison of ratios between arms using a paired t-test. As the distribution of ratios was highly skewed, the calculation was repeated using log ratios.

End of intervention and baseline breathlessness were related. As expected, a negative correlation between end-of-intervention minus baseline breathlessness and baseline breathlessness was seen. (fig. 1). Importantly, a uniform variability across all baseline intensities was seen with these absolute measures. This was not the case with ratio measures, where ratios became very large for lower intensity of breathlessness increasing the variability. Using log ratios decreased the variability.

Using data from the three placebo-controlled crossover trials $(N=95)$ [10-12], there were 113 evaluable preference responses from 93 participants to the question of which treatment arm gave the best benefit for
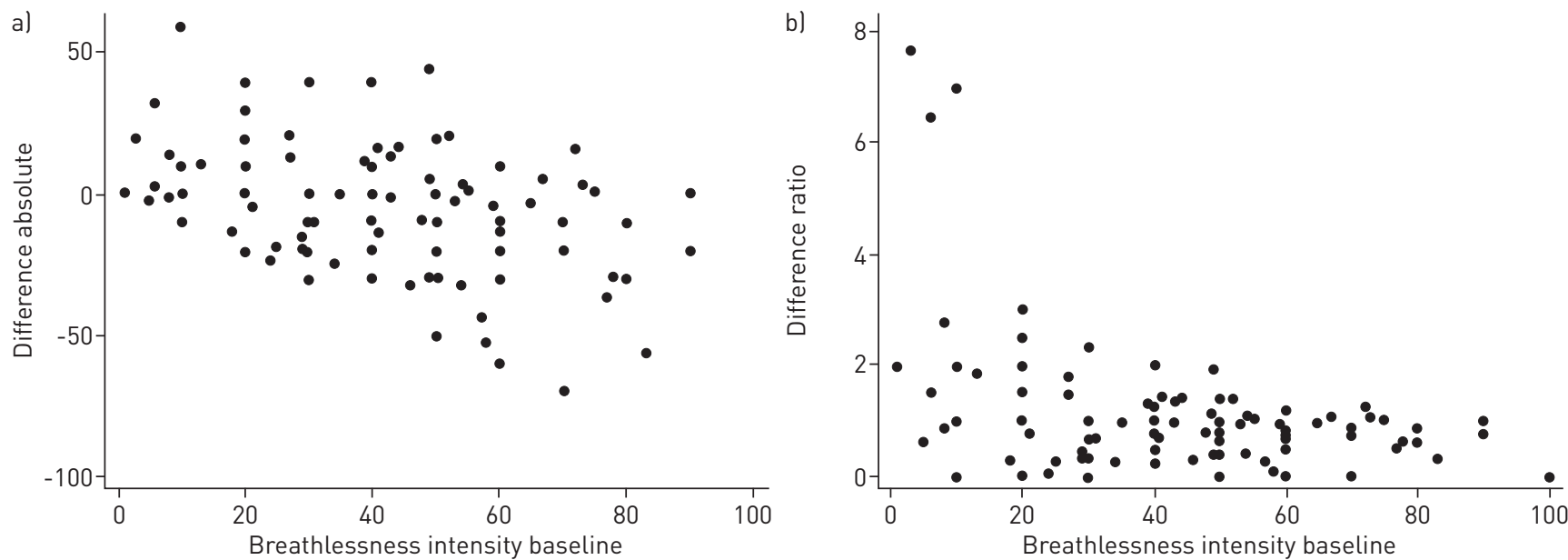

FIGURE 1 Variability against baseline intensity. The difference (end-of-treatment breathlessness intensity minus baseline measures) against baseline shown as absolute values has uniform variability when plotted against baseline intensity (a), whereas variability becomes very wide at lower intensities when values are plotted using ratios (b). 
their breathlessness. A preference was given in 93 of the 113 responses (drug $n=62$, placebo $n=33$, no preference $\mathrm{n}=18$ ). The mean ratio of breathlessness scores (end/baseline) in the preferred arm and combining all preferences (drug or placebo) was mean $\pm \mathrm{SD}, 0.87 \pm 0.87$. The mean ratio in the arm not chosen was $1.00 \pm 0.92$. There were 90 preferences where both a positive and a negative preference were stated. For these, a comparison of the ratios in the preferred and other arms showed a difference of -0.14 . Using log ratios, not including zero ratios, the comparison of the preferred and other arm ratios was a difference of -0.21 (after anti-log).

We believe that this is the first data-based demonstration that absolute measures of change in dyspnoea are preferable for study planning and evaluation of research results. There was uniform variation in response presented by change in absolute measures despite the relationship with baseline intensity, irrespective of baseline breathlessness intensity, which was not seen with breathlessness response expressed as a relative change.

When relative reductions are reported, using blinded patient preference as a patient anchor-based method, this study demonstrates that the MCID for the relative reduction should be $14 \%$ (using ratios) or $21 \%$ (using log ratios).

Eligibility criteria for entry to the studies contributing data to the pooled analysis resulted in fewer measures of "mild" breathlessness than for measures of $\geqslant 30 \mathrm{~mm}$. Thus results for mild baseline dyspnoea are less easily interpreted. However, our data show that variability of ratio measures becomes very large for low baseline breathlessness. This finding does not appear to support previous recommendations in the pain literature that in studies with no minimum baseline symptom intensity requirement clinical relevance should be defined in terms of relative change [13].

Results of clinical trials for chronic breathlessness should be presented as both absolute and relative measures since each differentially informs the interpretation of study results. The MCID expressed as a relative reduction is between $14 \%$ and $21 \%$.

However, there is uniform variation in response presented as absolute measures despite the relationship with baseline intensity, but not with relative measures. In view of this and because both distribution and patient anchor-based method can be used to calculate the MCID for absolute measures, we suggest that absolute measures should be used for the MCID in the calculation of sample sizes.

0 @ERSpublications

Dyspnoea trials should report absolute and relative values but use absolute measures for sample size calculation http://ow.ly/AuieS

Miriam J. Johnson ${ }^{1}$, J. Martin Bland ${ }^{2}$, Stephen G. Oxberry ${ }^{3}$, Amy P. Abernethy ${ }^{4,5}$ and David C. Currow ${ }^{5}$

${ }^{1}$ Hull York Medical School, University of Hull, Hull, UK. ${ }^{2}$ Dept of Health Sciences, University of York, York, UK.

${ }^{3}$ Kirkwood Hospice, Huddersfield, UK. ${ }^{4}$ Dept of Medicine, Duke University School of Medicine, Durham, NC, USA.

${ }^{5}$ Discipline of Palliative and Supportive Services, Flinders Centre for Clinical Change, Flinders University, Adelaide, Australia.

Correspondence: Miriam J. Johnson, Hertford Building, The University of Hull, Cottingham Road, Hull, HU6 7RX, UK. E-mail: miriam.johnson@hyms.ac.uk

Received: March 122014 | Accepted after revision: July 232014 | First published online: Sept 262014

Conflict of interest: Disclosures can be found alongside the online version of this article at erj.ersjournals.com

Acknowledgements: Thanks go to Tracey Hawkes (Scarborough Hospital, Scarborough, UK) for able assistance in combining the datasets.

\section{References}

Gigerenzer G, Edwards A. Simple tools for understanding risks: from innumeracy to insight. BMJ 2003; 327: 741-744.

Gigerenzer G, Wegwarth O, Feufel M. Misleading communication of risk. BMJ 2010; 341: c4830.

3 King NB, Harper S, Young ME. Use of relative and absolute effect measures in reporting health inequalities: structured review. BMJ 2012; 345: e5774.

4 Guyatt GH, Osoba D, Wu AW, et al. Methods to explain the clinical significance of health status measures. Mayo Clin Proc 2002; 77: 371-383.

Cohen J. A power primer. Psychol Bull 1992; 112: 155-159.

Dworkin RH, Turk DC, McDermott MP, et al. Interpreting the clinical importance of group differences in chronic pain clinical trials: IMMPACT recommendations. Pain 2009; 146: 238-244.

7 Currow DC, McDonald C, Oaten S, et al. Once-daily opioids for chronic dyspnea: a dose increment and pharmacovigilance study. J Pain Symptom. Manage 2011; 42: 388-399.

8 Galbraith S, Fagan P, Perkins P, et al. Does the use of a handheld fan improve chronic dyspnea? A randomized, controlled, crossover trial. J Pain Symptom. Manage 2010; 39: 831-838. 
9 Johnson MJ, Bland JM, Oxberry SG, et al. Clinically important differences in the intensity of chronic refractory breathlessness. J Pain Symptom Manage 2013; 46: 957-963.

10 Abernethy AP, Currow DC, Frith P, et al. Randomised, double blind, placebo controlled crossover trial of sustained release morphine for the management of refractory dyspnoea. BMJ 2003; 327: 523-528.

11 Johnson MJ, McDonagh TA, Harkness A, et al. Morphine for the relief of breathlessness in patients with chronic heart failure-a pilot study. Eur J Heart Fail 2002; 4: 753-756.

12 Oxberry SG, Torgerson DJ, Bland JM, et al. Short-term opioids for breathlessness in stable chronic heart failure: a randomized controlled trial. Eur J Heart Fail 2011; 13: 1006-1012.

13 Farrar JT, Young JP Jr, LaMoreaux L, et al. Clinical importance of changes in chronic pain intensity measured on an 11-point numerical pain rating scale. Pain 2001; 94: 149-158.

\title{
Rare $\alpha_{1}$-antitrypsin genotype in a grass seed worker
}

\author{
To the Editor:
}

We hereby present a case of chronic obstructive pulmonary disease (COPD) caused by a combination of occupational dust exposure and severe $\alpha_{1}$-antitrypsin $\left(\alpha_{1}\right.$-AT) deficiency. On evaluation of the genetics of this severe $\alpha_{1}$-AT deficiency, an apparent PiMM genotype was revealed to be heterozygotic for two different rare deficient alleles.

In 2008, a 62-year-old man was referred to Dept of Occupational Medicine, Aalborg University Hospital, Aalborg, Denmark, by his general practitioner (GP) to assess whether his reduced lung function was related to occupational dust exposure. For 26 years, he had been employed at a factory that produces grass seed, predominantly from rye grass. The factory received grass seeds from farmers in a dried condition, which were then cleaned and packed in bags for resale. Grass seeds were loaded as loose seeds, and mechanically cleaned, filtered and sorted. During the first 10-15 years of his employment, the process caused heavy dust development, especially when the grass seeds were cleaned but also when the cleaned seeds were weighed. From the early 1990s, the dust filters were improved on the cleaning machine, but still, a considerable dust exposure was reported. However, dust measurements were never performed. The staff did not wear dust masks or other protective equipment. In later years, grass seed handling was automated, including weighing and packing of seeds. The worker had part-time administrative work during the last few years, but he did spend a few hours in the plant every day. He continued working until his retirement in 2012. He had no other dust exposure and he was a social smoker until the mid-1980s, with a cumulative tobacco consumption of 3-4 pack-years. He had no prior history of asthma or other lung diseases. In 2000, he contacted his GP due to increasing exertional dyspnoea and an episode of near-syncope. He was hospitalised in the Dept of Cardiology without any abnormal findings. In 2002, radiography showed a flattened diaphragm. Lung function testing was carried out by the GP for the first time in 2008 and identified significant obstruction, and he was referred for an occupational health assessment. Besides dyspnoea, he showed no asthma symptoms, cough or expectoration, and no aggravation at work. On clinical examination, we observed slight exertional dyspnoea upon walking up one flight of stairs but no dyspnoea at rest. He had a hyperexpanded chest but normal auscultation. Lung function tests showed an obstructive pattern with forced vital capacity (FVC) $4.3 \mathrm{~L}$ (96\% predicted), forced expiratory volume in $1 \mathrm{~s}$ (FEV1) $1.9 \mathrm{~L}$ (55\% predicted) and FEV1/FVC ratio 0.43, and peak flow $252 \mathrm{~L} \cdot \mathrm{min}^{-1}$ (49\% predicted). Blood test showed a severely reduced $\alpha_{1}$-AT level $\left(0.13 \mathrm{~g} \cdot \mathrm{L}^{-1}\right.$, reference range $\left.0.9-2.0 \mathrm{~g} \cdot \mathrm{L}^{-1}\right)$. Skin-prick testing showed no signs of allergy. Genetic testing was performed first by looking for $\mathrm{Z}$ or $\mathrm{S}$ genotypes, but the patient was reported to be $\mathrm{Pi}^{\star} \mathrm{MM}$. Therefore, sequencing [1] was performed after DNA extraction from a dry blood spot. Final genotype was reported to be $\mathrm{Pi}^{\star} \mathrm{MmaltonMheerlen}$. He was reported to the Danish National Board of Industrial Injuries for workers' disability compensation for the COPD diagnosis and recognised as having a $20 \%$ disability. He was further referred to a lung department where he was treated with a combined inhaled corticosteroid and long-acting $\beta_{2}$-agonist and enrolled in a trial of substitution with human $\alpha_{1}$-AT versus placebo. He gave consent for publication as a case report.

A gene-environment interaction is a condition in which a genetic vulnerability in a subject in combination with harmful environmental factors causes illness or impairment of the individual. $\alpha_{1}$-AT deficiency has been suspected to be an inherited disorder of the lung since 1963 [2]. Before modern genetic tests were invented, gel electrophoresis was used to investigate the variants of $\alpha_{1}$-AT, characterised by M-, S- and 